\title{
Implications of the Collar Sign in Incompletely Occluded Aneurysms after Pipeline Embolization Device Implantation: A Follow-Up Study
}

\author{
(D) S. Gomez-Paz, (D)Y. Akamatsu, (D).M. Moore, (DC.S. Ogilvy, (D) A.J. Thomas, and (D) C.J. Griessenauer
}

\begin{abstract}
BACKGROUND AND PURPOSE: The angiographic collar sign has been recently described in patients with incompletely occluded aneurysms after Pipeline Embolization Device implantation. The long-term implications of this sign are unknown. We report angiographic outcomes of patients with the collar sign with follow-up of up to 45 months and the implications of this angiographic finding.
\end{abstract}

MATERIALS AND METHODS: We performed a retrospective review of a prospectively maintained data base of patients who underwent Pipeline Embolization Device implantation for an intracranial aneurysm at our institution between January 2014 and December 2016. We included patients with a collar sign at the initial follow-up angiogram after Pipeline Embolization Device implantation.

RESULTS: A total of 198 patients with 285 aneurysms were screened for the collar sign on initial and subsequent follow-up angiograms. There were 226 aneurysms (79.3\%) with complete occlusion at the first follow-up. Of 59 incompletely occluded aneurysms, 19 (32.2\%) aneurysms in 17 patients were found to have a collar sign on the first angiographic follow-up (median, 6 months; range, 4.2-7.2). Ten (52.6\%) aneurysms underwent retreatment with a second Pipeline Embolization Device, which resulted in aneurysm occlusion in $1(10 \%)$ patient. There were only $3(15.8 \%)$ aneurysms with complete occlusion at the last follow-up, 2 (10.5\%) of which had a single Pipeline Embolization Device implantation and another single (5.3\%) aneurysm with a second Pipeline Embolization Device implantation.

CONCLUSIONS: A collar sign on the initial angiogram after Pipeline Embolization Device placement is a predictor of poor aneurysm occlusion. Because the occlusion rates remain equally low regardless of retreatment in patients with a collar sign, radiologic followup may be more appropriate than retreatment.

ABBREVIATIONS: PED = Pipeline Embolization Device; ICAB = internal carotid artery bifurcation; $P O=$ paraophthalmic; PCOMM = posterior communicating artery

W e previously reported a series of incompletely occluded aneurysms after Pipeline Embolization Device (PED; Covidien, Irvine, California) implantation with an angiographic "collar sign," an unopacified white line between the parent vessel and the aneurysm dome on follow-up angiography. ${ }^{1}$ This finding was perceived to be indicative of near-yetincomplete endothelialization of the PED. The long-term

Received November 11, 2019; accepted after revision December 27.

From the Neurosurgical Service (S.G-P., Y.A., J.M.M., C.S.O., A.J.T.), Beth Israel Deaconess Medical Center, Harvard Medical School, Boston, Massachusetts; Department of Neurosurgery (C.J.G.), Geisinger, Danville, Pennsylvania; and Research Institute of Neurointervention (C.J.G.), Paracelsus Medical University, Salzburg, Austria.

Please address correspondence to Christoph J. Griessenauer, MD, Department of Neurosurgery, Geisinger, 100 N Academy Ave, Danville, PA 17822; e-mail: christoph.griessenauer@gmail.com; @SantiagoGP92; @cgriessenauer

Indicates article with supplemental on-line table.

http://dx.doi.org/10.3174/ajnr.A6415 implications of this finding are currently unknown. Here, we report the angiographic outcomes of patients with the collar sign with a follow-up of up to 45 months and the implications of this angiographic finding.

\section{MATERIALS AND METHODS}

After obtaining Institutional Review Board approval, we performed a retrospective review of a prospectively maintained data base of patients who underwent PED implantation for an intracranial aneurysm at Beth Israel Deaconess Medical Center between January 2014 and December 2016. We included patients with a collar sign at the initial follow-up angiogram after PED implantation. These radiographic images were obtained using the standard anteroposterior, lateral, and oblique digital subtraction angiography projections and were interpreted by 2 independent physicians with neuroendovascular training. Patients considered ineligible for this study were those without a collar sign on the 
first follow-up angiogram or without long-term follow-up of at least 1 year.

\section{RESULTS}

A total of 198 patients with 285 aneurysms treated with a PED between 2014 and 2016 were screened for the collar sign on initial and subsequent follow-up angiograms. There were 226 aneurysms (79.3\%) with complete occlusion at the first follow-up. Of 59 incompletely occluded aneurysms, 19 (32.2\%) aneurysms in 17 patients were found to have a collar sign on the first angiographic follow-up (median, 6 months; range, 4.2-7.2). Two patients had 2 separate aneurysms with a collar sign. Fifteen (78.9\%) aneurysms were paraophthalmic internal carotid artery aneurysms. Other locations included the middle cerebral artery (10.5\%), internal carotid artery bifurcation (5.3\%), and posterior communicating artery (5.3\%). There were 14 (73.7\%) aneurysms with branch vessels arising from the aneurysm dome. The median angiographic follow-up was 32.9 months (range, 11.9-45.2 months). Ten (52.6\%) aneurysms underwent retreatment with a second PED device, which resulted in aneurysm occlusion in $1(10 \%)$ aneurysm. The median time to retreatment was 15.8 months (range, 6.8-33.4 months). In terms of aneurysmal occlusion, there were $3(15.8 \%)$ aneurysms with complete occlusion at last follow-up, $2(10.5 \%)$ of which had a single PED implantation (time to occlusion, 11.9 and 42.2 months, respectively) and another single (5.3\%) aneurysm with a second PED implantation (time to occlusion, 12.1 months from the second PED implantation).

Among the $16(84.2 \%)$ aneurysms that did not occlude, a single $(5.3 \%)$ aneurysm with intraluminal thrombus enlarged (aneurysm 15), two (10.5\%) aneurysms did not change in size, and 13 (68.4\%) decreased in size compared with the initial angiogram. Satisfactory wall apposition as demonstrated on fluoroscopy was achieved in all cases. There were no procedural complications or neurologic long-term sequelae. A full summary of patient characteristics and follow-up results, categorized in 4 groups based on occlusion status and retreatment, is shown in the On-line Table. A representative set of images from each of the 4 groups is shown in the Figure.

\section{DISCUSSION}

We report the angiographic outcomes of incompletely occluded intracranial aneurysms with a collar sign following PED implantation for up to 45 months. We observed a collar sign in $32.2 \%$ of incompletely occluded aneurysms at first follow-up. Of 19 aneurysms with a collar sign, complete occlusion was achieved in only $3(15.8 \%)$ aneurysms, including 2 aneurysms that spontaneously occluded after initial treatment and 1 aneurysm that occluded after retreatment with a second PED. However, we observed an incomplete occlusion with a persistent collar sign in $16(84.2 \%)$ aneurysms, even after a second or third PED implantation. Aneurysm enlargement was observed in a single large thrombosed aneurysm. More than two-thirds of incompletely occluded aneurysms decreased in size, and none ruptured during follow-up.

\section{Endothelialization of the Pipeline Flow Diverter}

As described in preclinical studies, endothelialization along the stent strut of the PED has been identified as the key underlying mechanism of aneurysmal exclusion from the intracranial circulation and subsequent aneurysm occlusion. ${ }^{2-6}$ Likewise, this mechanism of endothelialization along the PED strut has been confirmed in humans. ${ }^{1,7}$ Initially, the collar sign was thought to be indicative of future aneurysmal occlusion following PED implantation. Nonetheless, our results demonstrate that this sign is likely a sign of persistent aneurysmal flow even at long-term follow-up, regardless of any additional PED implantation.

Although the underlying mechanism is still unknown, we found branch arteries originating from the aneurysm dome in 14 of 19 cases (73.7\%). On the basis of this finding, we hypothesized that an aspiration effect led by a pressure gradient between the parent arteries and branches is the likely cause of inhibition of complete endothelialization along the PED strut and subsequent persistence of flow into the branches through the aneurysm. Two of 3 aneurysms with complete occlusion did not have a branch arising from the aneurysm at the initial follow-up angiogram, which further supports this hypothesis. Furthermore, patient 1 had an ophthalmic artery that arose from the aneurysm dome, which had been opacified at the first follow-up angiogram. Subsequently, both the aneurysm and the ophthalmic artery were found to be occluded on the final follow-up angiogram without any visual sequelae, suggesting collateral compensation to the distal segment of the ophthalmic artery and depletion of aspiration effect. Therefore, the collar sign is likely an indication of partial endothelialization, seen more commonly in aneurysms with a branch arising from the dome, which demands persistent blood supply and subsequently inhibits complete endothelialization required to cover the aneurysm neck. A negative correlation between aneurysm occlusion and branch vessels arising from the aneurysm neck or dome following PED implantation in patients with paraophthalmic artery aneurysms has been reported. ${ }^{8}$

On the other hand, the collar sign was observed in a thrombosed aneurysm without an associated branch vessel (aneurysm 15). In the case of thrombosed aneurysms, reports have described a heterogeneous thrombosis status, in which both thrombotic and thrombolytic processes take place inside the aneurysm. These result from a cycle of repeat adventitial inflammation, capillary proliferation, and bleeding within the aneurysmal wall in such thrombosed aneurysms. ${ }^{9,10}$ Therefore, endothelialization along the aneurysm neck is less likely to occur following PED implantation.

\section{Management Strategies for Incompletely Occluded Aneurysms with the Collar Sign}

In the present study, complete occlusion was achieved in only 3 aneurysms (15.8\%) with the collar sign. Aneurysm enlargement was observed in only 1 patient with a thrombosed aneurysm as mentioned previously. In contrast, most of the aneurysms with a collar sign were stable or even decreased in size $(10.5 \%$ and $68.4 \%$, respectively), suggesting a stabilizing effect of the PEDs on the aneurysm. Furthermore, because the rates of complete occlusion of the aneurysm were very low regardless of the number of 


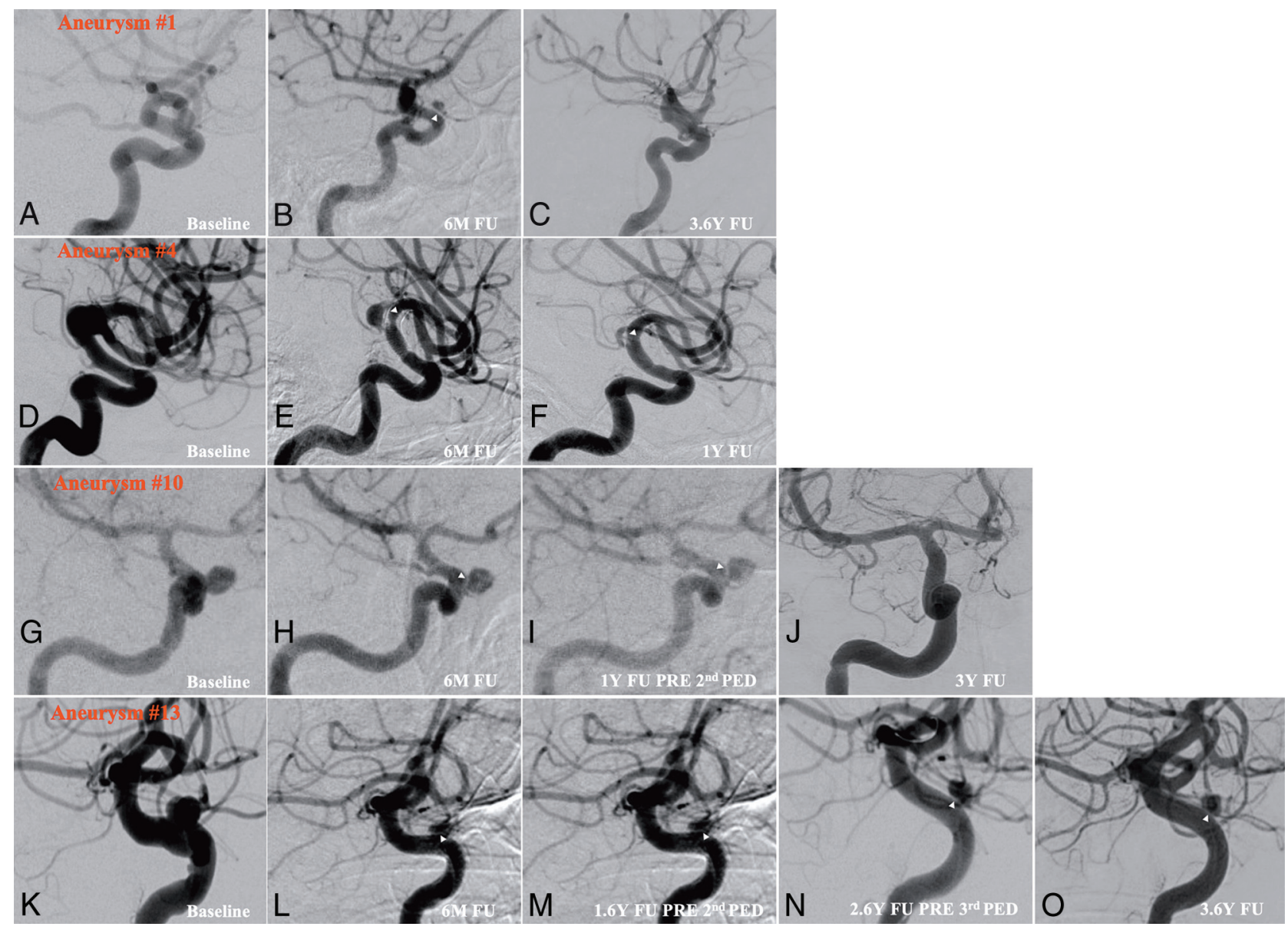

FIGURE. Digital subtraction angiograms showing the collar sign in incompletely occluded aneurysms after Pipeline embolization. Each row represents 1 aneurysm from each group corresponding to the On-line Table. The first column (far-left) shows the aneurysm before Pipeline embolization. The second column shows the residual aneurysm with the collar sign (white arrowheads) after Pipeline placement at initial follow-up. Further columns (3-5) are based on the patient group (eg, spontaneous occlusion during follow-up, residual filling and collar sign at last follow-up without intervention, occlusion during follow-up after a 2nd PED, and residual filling and collar sign at last follow-up after a 2nd PED). Aneurysm 1 for row 1: baseline, first follow-up, and last angiographic followup are shown. Aneurysm 4 for row 2: baseline, first follow-up, and last angiographic follow-up are shown. Aneurysm 10 for row 3: baseline, first follow-up, before the second PED, and last angiographic follow-up are shown. Aneurysm 13 for row 4: baseline, first follow-up, before the second PED, before the third PED, and last angiographic follow-up are shown. M indicates month; Y, year; FU, follow-up.

PED treatments, retreatment with adjunctive PED implantation is unlikely to be effective in aneurysms with a collar sign. Under these circumstances, radiologic follow-up may be more appropriate than retreatment. Thus, patients are not committed to the risk of an additional procedure in the absence of the expected benefit of aneurysm occlusion.

\section{CONCLUSIONS}

The collar sign is indicative of incomplete endothelialization and persistent aneurysmal flow even during medium-to-long-term follow-up periods. A collar sign on the initial angiogram after PED placement is therefore a predictor of poor aneurysm occlusion. Because the occlusion rates remain equally low regardless of retreatment in patients with a collar sign, radiologic follow-up may be more appropriate than retreatment. Thus, patients are not exposed to the risk of an additional procedure in the absence of the expected benefit of aneurysm occlusion. This study provides some additional evidence that aneurysms with branches arising from the dome may not be suitable for flow diversion.

Disclosures: Ajith J. Thomas_UNRELATED: Consultancy: Stryker, Comments: Data Safety Monitoring Board*; Expert Testimony: CRICO, Comments: cerebrovascular disorders; Royalties: Primer of Cerebrovascular Disorders. *Money paid to the institution.

\section{REFERENCES}

1. Griessenauer CJ, Gupta R, Shi S, et al. Collar sign in incompletely occluded aneurysms after Pipeline Embolization: evaluation with angiography and optical coherence tomography. AJNR Am J Neuroradiol 2017;38:323-26 CrossRef Medline

2. Kadirvel R, Ding YH, Dai D, et al. Cellular mechanisms of aneurysm occlusion after treatment with a flow diverter. Radiology 2014; 270:394-99 CrossRef Medline

3. Ding Y, Dai D, Kallmes DF, et al. Preclinical testing of a novel thin film nitinol flow-diversion stent in a rabbit elastase aneurysm model. AJNR Am J Neuroradiol 2016;37:497-501 CrossRef Medline 
4. Marosfoi M, Langan ET, Strittmatter L, et al. In situ tissue engineering: endothelial growth patterns as a function of flow diverter design. J Neurointerv Surg 2017;9:994-98 CrossRef Medline

5. Matsuda Y, Chung J, Lopes DK. Analysis of neointima development in flow diverters using optical coherence tomography imaging. $J$ Neurointerv Surg 2018;10:162-67 CrossRef Medline

6. Rouchaud A, Ramana C, Brinjikji W, et al. Wall apposition is a key factor for aneurysm occlusion after flow diversion: a histologic evaluation in 41 rabbits. AJNR Am J Neuroradiol 2016;37:2087-91 CrossRef Medline

7. Szikora I, Turanyi E, Marosfoi M. Evolution of flow-diverter endothelialization and thrombus organization in giant fusiform aneurysms after flow diversion: a histopathologic study. AJNR Am J Neuroradiol 2015;36:1716-20 CrossRef Medline

8. Griessenauer CJ, Ogilvy CS, Foreman PM, et al. Pipeline Embolization Device for small paraophthalmic artery aneurysms with an emphasis on the anatomical relationship of ophthalmic artery origin and aneurysm. J Neurosurg 2016;125:1352-59 CrossRef Medline

9. Yasui T, Sakamoto H, Kishi H, et al. Rupture mechanism of a thrombosed slow-growing giant aneurysm of the vertebral artery-case report. Neurol Med Chir (Tokyo) 1998;38:860-64 CrossRef Medline

10. Nagahiro S, Takada A, Goto $S$, et al. Thrombosed growing giant aneurysms of the vertebral artery: growth mechanism and management. J Neurosurg 1995;82:796-801 CrossRef Medline 